\title{
Habitual variable sleep and Type A behavior
}

\author{
ROBERT A. HICKS, SCOTT LINGEN, and PAT COLLINSWORTH EASTMAN \\ San Jose State University, San Jose, California 95192
}

\begin{abstract}
The responses to the Jenkins Activity Survey of groups of $\mathbf{4 0}$ short sleepers, $\mathbf{4 0}$ long sleepers, and 40 variable sleepers were compared. As predicted, it was found that the variable sleepers showed significantly less Type A behavior than either of the other two groups. These data further elaborate the possible role of sleep behavior in the management of stress.
\end{abstract}

Recently, the San Jose group (Hicks, Pellegrini, Martin, Garbesi, Elliott, \& Hawkins, 1979) reported finding a significant inverse relationship between normal habitual sleep duration and level of Type A behavior as defined by score on the Jenkins Activity Survey (JAS). In discussing the implications of this relationship, we speculated that the relatively higher stress susceptibility of Type A individuals might, in part, be a consequence of the relatively shorter sleep durations that tend to be characteristic of these individuals. That is, the Type A person tends to adopt the habit of sleeping less, which in turn may reduce the amount of REM sleep but leave intact the deeper stages (3 and 4) of NREM sleep.

In this regard, Hartmann and Brewer (1976) demonstrated that the sleep duration of a group of variable sleepers tended to fluctuate as a function of the stressfulness of their life situations. Specifically, their variable sleepers reported needing more sleep during periods of increased "mental or emotional stress." In commenting on this result, Hartmann and Brewer suggested that it was probably the requirement for REM sleep that changes as a function of the need to resolve worry or stress and, hence, the need to change the duration of sleep.

While the results of Hartmann and Brewer's (1976) study are not incongruent with the aforementioned inverse relationship between normal habitual sleep duration and score on the JAS, their data suggest a possible qualification of that relationship. To elaborate, Hicks et al. (1979) systematically excluded self-reported variable sleepers from consideration as subjects. Thus, their results may be somewhat misleading in the sense that stable sleep duration was emphasized as the sleep factor that seemed to be implicated in the formation of Type A behavior. Clearly, Hartmann and Brewer's (1976) comments suggest that, because of their ability to adjust sleep appropriately to the stressfulness of life, variable sleepers should show less Type A behavior than nonvariable sleepers, regardless of mean sleep duration.

Therefore, the purpose of this research was to deter-

We are grateful to Signe Gary for her help in preparing this report. Requests for reprints should be addressed to Robert A. Hicks, Department of Psychology, San Jose State University, San Jose, California 95192. mine the relationship between habitual variable sleep and level of Type A behavior. Congruent with the foregoing discussion, we predicted that a randomly selected group of variable sleepers would score lower on the JAS than nonvariable groups of either short or long sleepers.

\section{METHOD}

To test the hypothesis, more than 1,000 college students were given a form of the JAS modified for college students (Glass, 1977) and a self-report sleep questionnaire (Hicks \& Pellegrini, 1977). To assess normal habitual sleep pattern, the following questions were asked: "How many hours do you sleep each night?" "How long have you been experiencing this duration of sleep?" "Are you satisfied with the amount of sleep you get?" We excluded from further consideration those students who had experienced a given pattern of sleep for less than 1 year or who were dissatisfied with their sleep. (For a discussion that supports the need to control these parameters, see Cartwright, 1978.) Next, from appropriate pools of eligible individuals, three sleep groups were randomly selected: a group of 20 male and 20 female variable sleepers (defined as persons who reported at least a 2-h range in their regular duration of sleep), a group of 20 male and 20 female short sleepers (defined as persons who slept, without reported variation, $6 \mathrm{~h}$ or less each night), and a group of 20 male and 20 female long sleepers (defined as persons who slept, without reported variation, $9 \mathrm{~h}$ or more each night). It should be noted that the two extreme sleep groups were included as a means of duplicating the extremes of the continuum used by Hicks et al. (1979). Thus, these groups provided the reference points that were necessary to tie the results of this research to that study. The JAS was scored using a key that was generously given to us by David C. Glass.

\section{RESULTS AND DISCUSSION}

First, we computed the mean JAS score and the standard deviation for the variable, short, and long sleeper groups. These values were $6.4 \pm 2.9,9.4 \pm 2.5$, and $7.6 \pm 3.0$, respectively. Next we analyzed the data with a 3 (sleep groups) by 2 (sex) factor analysis of variance. The results of this ANOVA revealed a significant effect for sleep groups $[\mathrm{F}(2,114)=9.10, \mathrm{p}<.01]$ and nonsignificant sex and Sleep Group by Sex effects $[F(1,114)=.22$, and $F(2,114)=1.14$, respectively $]$. (Because we found no sex effects, we have reported means and the standard deviations derived from all of the subjects in each of the sleep groups.) Finally, we 
tested the differences between pairs of individual group means and found, as was predicted, that the variable sleepers scored significantly lower on the JAS than did the long sleepers $[t(78)=1.85, p<.05$, one-tailed] and that the long sleepers scored significantly lower on the JAS than did the short sleepers $[t(78)=2.90, p<.01$, one-tailed]. (The latter of these two comparisons simply serves to replicate the inverse relationship between score on the JAS and sleep duration that we cited earlier.)

The relationship set forth by these data suggests that variable sleep may prove to be a more important correlate oi level of Type A behavior than sleep duration. Certainly, this is a parameter of habitual sleep pattern that merits additional attention. In this regard, it is worth noting that most college students do not give selfreports that would lead us to categorize them as habitual variable sleepers. It may prove to be the case that the value of varying sleep duration to cope with stress must be learned. Further, it may also be true that, once he learns this value, an individual must enjoy a lifestyle that permits the opportunity for longer periods of sleep during times of stress. Perhaps the Type A person either is one who has not learned the value of sleep as a stress coping mechanism and/or is a person who has adopted a schedule of wakeful activity that precludes the possibility of flexible sleep durations during periods of stress. This is a relationship that should be elaborated further because the possibility exists that the management of sleep habits may prove to be a useful adjunct to successfully modifying Type A behavior.

\section{REFERENCES}

Cartwright, R. D. A primer on sleep and dreaming. Reading, Mass: Addison-Wesley, 1978.

Glass, D. C. Behavior patterns, stress, and coronary disease. Hillsdale, N.J: Erlbaum, 1977.

Hartmann, E., \& Brewer, J. When is more or less sleep required? A study of variable sleepers. Comprehensive Psychiatry, 1976, 17, $275-284$.

Hicks, R. A., \& Pellegrini, R. J. Anxiety levels of short and long sleepers. Psychological Reports, 1977, 41, 569-570.

Hicks, R. A., Pellegrini, R. J., Martin, S., Garbesi, L., Elliotr, D., \& Hawkins, J. Type A behavior and normal habitual sleep duration. Bulletin of the Psychonomic Society, $1979,14,185-186$.

(Received for publication October 22, 1979.) 